\title{
Locating Equitable Domination and Independence Subdivision Numbers of Graphs
}

\author{
P.Sumathi ${ }^{1}$, G.Alarmelumangai ${ }^{2}$ \\ ${ }^{1}$ Head \& Associate Professor of Mathematics, C.K.N college for Men, Anna Nagar, \\ Chennai-600102. \\ ${ }^{2}$ Assistant professor of Mathematics, E.M.G Yadava women's college, \\ Madurai-625014
}

Keywords: Locating equitable domination, Independence, Subdivision numbers.

\begin{abstract}
Let $\mathrm{G}=(\mathrm{V}, \mathrm{E})$ be a simple, undirected, finite nontrivial graph. A non empty set $\mathrm{D} \subseteq \mathrm{V}$ of vertices in a graph $\mathrm{G}$ is a dominating set if every vertex in $\mathrm{V}$-D is adjacent to some vertex in D. The domination number $\gamma(\mathrm{G})$ is the minimum cardinality of a dominating set of $\mathrm{G}$. A dominating set $\mathrm{D}$ is a locating equitable dominating set of $\mathrm{G}$ if for any two vertices $\mathrm{u}, \mathrm{w} \in \mathrm{V}$ $\mathrm{D}, \mathrm{N}(\mathrm{u}) \cap \mathrm{D} \neq \mathrm{N}(\mathrm{w}) \cap \mathrm{D},|\mathrm{N}(\mathrm{u}) \cap \mathrm{D}|=|\mathrm{N}(\mathrm{w}) \cap \mathrm{D}|$. The locating equitable domination number of $\mathrm{G}$ is the minimum cardinality of a locating equitable dominating set of $G$. The locating equitable domination subdivision number of $G$ is the minimum number of edges that must be subdivided(where each edge in $\mathrm{G}$ can be subdivided at most once) in order to increase the locating equitable domination number and is denoted by $\operatorname{sd} \gamma \mathrm{le}(\mathrm{G})$. The independence subdivision number $\operatorname{sd} \beta$ le $(G)$ to equal the minimum number of edges that must be subdivided in order to increase the independence number. In this paper, we establish bounds on $\operatorname{sd} \gamma l e(G)$ and $\operatorname{sd} \beta$ le $(G)$ for some families of graphs.
\end{abstract}

\section{Introduction}

For notation and graph theory terminology, we in general follow [3]. Specifically, a graph $G$ is a finite nonempty set $\mathrm{V}(\mathrm{G})$ of objects called vertices together with a possibly empty set $E(G)$ of 2element subsets of $V(G)$ called edges. The order of $G$ is $n(G)=|V(G)|$ and the size of $G$ is $m(G)=$ $|E(G)|$. The degree of a vertex $v \in V(G)$ in $G$ is $d G(v)=\left|N_{G}(v)\right|$. A vertex of degree one is called an end-vertex. The minimum and maximum degree among the vertices of $\mathrm{G}$ is denoted by $\delta(\mathrm{G})$ and $\Delta(\mathrm{G})$, respectively. Further for a subset $\mathrm{S} \subseteq \mathrm{V}(\mathrm{G})$, the degree of $\mathrm{v}$ in $\mathrm{S}$, denoted ds(v), is the number of vertices in $\mathrm{S}$ adjacent to $\mathrm{v}$; that is, $\mathrm{d}_{\mathrm{S}}(\mathrm{v})=|\mathrm{N}(\mathrm{v}) \cap \mathrm{S}|$. In particular, $\mathrm{d}_{\mathrm{G}}(\mathrm{v})=\mathrm{d}_{\mathrm{V}}(\mathrm{v})$. if the graph $\mathrm{G}$ is clear from the context, we simply write $\mathrm{V}, \mathrm{E}, \mathrm{n}, \mathrm{m}, \mathrm{d}(\mathrm{v}), \delta$ and $\Delta$ rather than $\mathrm{V}(\mathrm{G}), \mathrm{E}(\mathrm{G}), \mathrm{n}(\mathrm{G}), \mathrm{m}(\mathrm{G}), \mathrm{d} \mathrm{G}(\mathrm{v}), \delta(\mathrm{G})$ and $\Delta(\mathrm{G})$, respectively.

The closed neighborhood of a vertex $\mathrm{u} \in \mathrm{V}$ is the set $\mathrm{N}[\mathrm{u}]=\{\mathrm{u}\} \cup\{\mathrm{v} / \mathrm{uv} \in\}$. Given a set $\mathrm{S} \subseteq \mathrm{V}$ of vertices and a vertex $u \in S$, the private neighbor set of $u$, with respect to $S$, is the set $p n[n, S]=$ $\mathrm{N}[\mathrm{u}]-\mathrm{N}[\mathrm{S}-\{\mathrm{u}\}]$. We say that every vertex $\mathrm{v} \in \mathrm{pn}[\mathrm{u}, \mathrm{S}]$ is a private neighbor of $\mathrm{u}$ with respect to $\mathrm{S}$. Such a vertex $\mathrm{v}$ is adjacent to $\mathrm{u}$ but is not adjacent to any other vertex of $\mathrm{S}$, then it is an isolated vertex in the subgraph $\mathrm{G}[\mathrm{S}]$ induced by $\mathrm{S}$. In this case, $\mathrm{u} \in \mathrm{pn}[\mathrm{u}, \mathrm{S}]$, and we say that $\mathrm{u}$ is its own private neighbor. We note that if a set $\mathrm{s}$ is a $\gamma(\mathrm{G})$-set, then for every vertex $\mathrm{u} \in \mathrm{S}, \mathrm{pn}[\mathrm{u}, \mathrm{S}] \neq \varphi$, i.e., every vertex of $S$ has at least one private neighbor. It can be seen that if $S$ is a $\gamma(G)$-set, and two vertices $u, v \in S$ are adjacent, then each of $u$ and $v$ must have a private neighbor other than itself. We will slao use the following terminology. Let $v \in \mathrm{V}$ be a vertex of degree one; $v$ is called a leaf. The only vertex adjacent to a leaf, say $u$, is called a support vertex, and the edge uv is called a pendant edge.Two edges in a graph $\mathrm{G}$ are independent if they are not adjacent in $\mathrm{G}$. The distance $d_{G}(u, v)$ or $d(u, v)$ between two vertices $u$ and $v$ in a graph $G$, is the length of a shortest path connecting $\mathrm{u}$ and $\mathrm{v}$. The diameter of a connected graph $\mathrm{G}$ is defined to be $\max \{\mathrm{dG}(\mathrm{u}, \mathrm{v})$ $: \mathrm{u}, \mathrm{V} \in \mathrm{V}(\mathrm{G})\}$. A set $\mathrm{D} \subseteq \mathrm{V}$ of vertices is a dominating set if every vertex in $\mathrm{V}$-D is adjacent to 
some vertex in D. The domination number $\gamma(\mathrm{G})$ is the minimum cardinality of a dominating set of $\mathrm{G}$.

The locating equitable domination subdivision number of a graph $G$, denoted by $\operatorname{sd} \gamma 1 \mathrm{e}(\mathrm{G})$, equals the minimum number of edges that must be subdivided in order to create a graph $G$ ' for which $\gamma \mathrm{le}\left(\mathrm{G}^{\prime}\right)>\gamma_{\mathrm{le}}(\mathrm{G})$. The independence number $\beta(\mathrm{G})$ is the maximum cardinality of an independent set in G.we call an independent set $S$ of cardinality $\beta(G)$ a $\beta(G)$-set. The locating equitable independence subdivision number $\operatorname{sd} \beta$ le $(G)$ to equal the minimum number of edges that must be subdivided in order to create a graph $G^{\prime}$ for which $\beta$ le $\left(G^{\prime}\right)>\beta$ le $(G)$.

Results on the locating equitabdomination and independence subdivision numbers are given in sections 2 and 3 respectively.

Example 1.The following is an example of a graph whose $\operatorname{sdgle}(\mathrm{G})=1$.

Let $\mathrm{G}=(\mathrm{V}, \mathrm{E})$

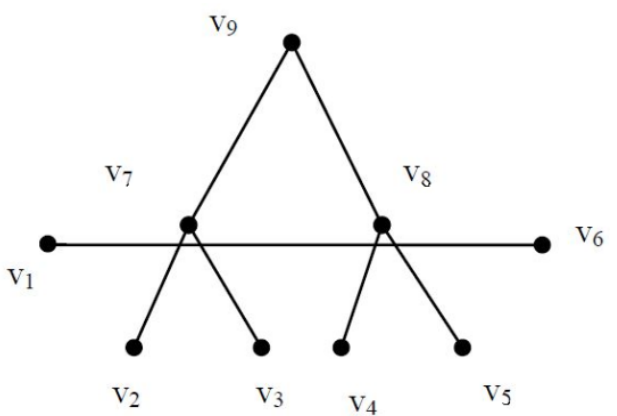

$\mathrm{V}=\{\mathrm{v} 1, \mathrm{v} 2, \mathrm{v} 3, \mathrm{v} 4, \mathrm{v} 5, \mathrm{v} 6, \mathrm{v} 7, \mathrm{v} 8, \mathrm{v} 9\}$,

$\mathrm{D}=\{\mathrm{v} 1, \mathrm{v} 2, \mathrm{v} 3, \mathrm{v} 4, \mathrm{v} 5, \mathrm{v} 6, \mathrm{v} 9\}$ is a $\gamma l \mathrm{e}^{-} \mathrm{set}, \quad \gamma \mathrm{le}(\mathrm{G})=7$.

After making a single subdivision in $\mathrm{G}$, the number $\gamma \mathrm{le}$ will be change. If we subdivide the edge v7v8 then $\gamma$ le increases. Hence $\operatorname{sd} \gamma$ le $(G)=1$.

\section{Results on the Locating Equitable Domination Subdivision numbers}

(i) $\operatorname{sd} \gamma l e\left(K_{n}\right)=2$

(ii) $\operatorname{sd} \gamma \operatorname{le}\left(P_{n}\right)=\left\{\begin{array}{l}1 \text { if } n \text { is odd } \\ 2 \text { if } n \text { is even }\end{array}\right.$

(iii) $\operatorname{sd} \gamma \operatorname{le}\left(C_{n}\right)=\left\{\begin{array}{l}1 \text { if } n \text { is even } \\ 2 \text { if } n \text { is odd }\end{array}\right.$

Proposition 2.1. For any tree $T$ of order $n \geq 3,1 \leq \operatorname{sd} \gamma l e(T) \leq 3$.

Proposition 2.2. For any k-regular graph $\mathrm{G}$ where $\mathrm{k} \geq 2, \quad 1 \leq \operatorname{sd} \gamma \mathrm{le}(\mathrm{G}) \leq 3$.

Theorem 2.3. For any connected graph $G$ of order $n \geq 3$, and for any two adjacent vertices $u$ and $\mathrm{v}$, where $\operatorname{deg}(\mathrm{u}) \geq 2$ and $\operatorname{deg}(\mathrm{v}) \geq 2, \quad \operatorname{sd} \gamma \operatorname{le}(\mathrm{G}) \leq \operatorname{deg}(\mathrm{u})+\operatorname{deg}(\mathrm{v})-1$.

Proof. Let uv be an edge in $G$, and let $G$ ' be the graph which results from subdividing all edges incident to $\mathrm{u}$ and $\mathrm{v}$. Thus, $\operatorname{deg}(\mathrm{u})+\operatorname{deg}(\mathrm{v})-1$ edges will be subdivided. We assume that both $\operatorname{deg}(\mathrm{u})$ $\geq 2$ and $\operatorname{deg}(\mathrm{v}) \geq 2$. We will show that $\gamma \mathrm{le}\left(\mathrm{G}^{\prime}\right)>\gamma_{\mathrm{le}}(\mathrm{G})$ by showing that (I) no $\gamma \mathrm{le}(\mathrm{G})$-set is also locating equitable dominating set of $G^{\prime}$ and (II) there is no locating equitable dominating set of $G^{\prime}$ of cardinality $\gamma(\mathrm{G})$ that contains a subdivision vertex. 
(i) Let $\mathrm{D}$ be an arbitrary $\gamma \mathrm{le}(\mathrm{G})$-set.We will show that $\mathrm{D}$ is not a locating equitable dominating set of G'.

Caes (1). $u, v \in D$. In this case, both $u$ and $v$ must have private neighbors other than themselves.But then neither $u$ nor $v$ dominate locating equitably these private neighbors in G'. Case(2). Either $u \notin D$ or $\mathrm{v} \notin \mathrm{D}$. in this case, $\mathrm{D}$ no longer locating equitable dominates $\{\mathrm{u}, \mathrm{v}\} \cap(\mathrm{V}-\mathrm{D})$ in $\mathrm{G}$ '.

(ii) Let $D$ be a subset of $G^{\prime}$ of cardinality $\gamma / \mathrm{l}(\mathrm{G})$ which contains at least one subdivision vertex. We will show that $D$ is not a locating equitable dominating set of $G$ '.

Assume to the contrary that G' contains a locating equitable dominating set of cardinality $\gamma_{\mathrm{le}}(\mathrm{G})$ which contains at least one subdivision vertex.Among all such dominating sets, let $\mathrm{D}^{*}$ be one which contains a minimum number of subdivision vertices. Assume, without loss of generality, that $\mathrm{D}^{*}$ contains a subdivision vertex adjacent to $\mathrm{v}$, call it $\mathrm{v}$, which subdivides the edge $\mathrm{vw}(\mathrm{w} \neq \mathrm{u})$.

It follows that $\mathrm{v} \notin \mathrm{D}^{*}$, since if $\mathrm{v} \in \mathrm{D}^{*}$, then $\mathrm{D}=\mathrm{D}^{*}-\left\{\mathrm{v}^{\prime}\right\} \cup\{\mathrm{w}\}$ is a locating equitable dominating set of $\mathrm{G}^{\prime}$ of cardinality $\gamma \mathrm{le}(\mathrm{G})$ contains fewer subdivision vertices than $\mathrm{D}^{*}$, contradicting the minimality of $\mathrm{D}^{*}$.

Clearly, v' can only be used to locating equitable dominate vertices v, v' and w. It follows that no other subdivision vertex adjacent to $\mathrm{v}$ is in $\mathrm{D}^{*}$, since any such vertices could be exchanced with their neighbors not equal to $\mathrm{v}$, to create a locating equitable dominating set of the same cardinality with fewer subdivision vertices, again contradicting the minimality of $\mathrm{D}^{*}$. It follows, therefore, that $\mathrm{u} \in \mathrm{D}^{*}$ since $\mathrm{D}^{*}$ is a dominating set and $\mathrm{u}$ is the only vertex available to locating equitable dominate the subdivision vertex, say $\mathrm{x}$, between $\mathrm{u}$ and $\mathrm{v}$, and $\mathrm{x} \notin \mathrm{D}^{*}$. Then no subdivision vertex adjacent to $\mathrm{u}$ is in $\mathrm{D}^{*}$, since $\mathrm{x} \notin \mathrm{D}^{*}$ and any other such vertex can be exchanged with its neighbor with fewer subdivision vertices than $\mathrm{D}^{*}$, again contradicting the minimality of $\mathrm{D}^{*}$.

At this pont we have established that (i) $\mathrm{v} \notin \mathrm{D}^{*}$, (ii) $\mathrm{u}, \mathrm{v}^{\prime} \in \mathrm{D}^{*}$, and (iii) every neighbor of $\mathrm{v}$ in $\mathrm{G}$ other than $\mathrm{w}$ is in $\mathrm{D}^{*}$, since the subdivision vertices adjacent to $\mathrm{v}$ are not in $\mathrm{D}^{*}$ and must be locating equitable dominated. In fact, $\mathrm{D}^{*}$ contains only one subdivision vertex, namely v'.But if $\mathrm{D}^{*}$ is a locating equitable dominating set of $\mathrm{G}^{\prime}$ of cardinality $\gamma \mathrm{le}(\mathrm{G})$, then it follows that $\mathrm{D}$ $=\mathrm{D}^{*}-\left\{\mathrm{u}, \mathrm{v}^{\prime}\right\} \cup\{\mathrm{v}\}$ is a dominating set of $\mathrm{G}$ of cardinality less than $\gamma \mathrm{le}(\mathrm{G})$, a contradiction. (This follows from the observation that $v^{\prime}$ is only needed to dominate vertices $v, v^{\prime} w$ in $G^{\prime}$, and $u$ is only needed to dominate itself and the subdivision vertices adjacent to it in G'). Earlier in the proof, we assumed that $\mathrm{D}^{*}$ contains a subdivision vertex adjacent tov, call it $\mathrm{v}$, which subdivides the edge $\mathrm{vw}(\mathrm{u} \neq \mathrm{w})$. It remains to consider the final case that $\mathrm{D}^{*}$ contains the subdivision vertex $\mathrm{x}$ between vertices $\mathrm{u}$ and $\mathrm{v}$.In this case we can assume that $\mathrm{D}^{*}$ contains no other subdivision vertex, Otherwise, they could be exchanged, as before, with their neighbors not equal to either $\mathrm{u}$ or $\mathrm{v}$, to produce a dominating set of the same cardinality but with fewer subdivision vertices, contradicting the minmimality of $D^{*}$. but vertex $x$ can only be used to dominate vertices $\mathrm{u}, \mathrm{x}$ andv, which means that $\mathrm{D}^{*}$ cannot contain both $\mathrm{u}$ and $\mathrm{v}$ (else vertex $\mathrm{x}$ is not needed). Therefore there are only three cases to consider.

Case 1. $\mathrm{u} \in \mathrm{D}^{*}$ and $\mathrm{v} \notin \mathrm{D}^{*}$.

Case2. $\mathrm{u} \notin \mathrm{D}^{*}$ and $\mathrm{v} \in \mathrm{D}^{*}$.

Case $3 . \mathrm{u} \notin \mathrm{D}^{*}$ and $\mathrm{v} \notin \mathrm{D}^{*}$.

But in each of the first two cases, it can be seen that the set $D^{*}-\{x\}$ is a locating equitable dominating set of $\mathrm{G}$ of cardinality less than $\gamma \mathrm{le}(\mathrm{G})$, a contradiction. In case 3, since $\operatorname{deg}(\mathrm{u}) \geq 2$ and $\operatorname{deg}(\mathrm{v}) \geq 2$, then $\mathrm{D}^{*_{-}}\{\mathrm{x}\}$ is a locating equitable dominating set of $\mathrm{G}$ of cardinality less than $\gamma l e(G)$, since every neighbor of $u$ or $v$ in $G$,other than $u$ and $v$, is in $D^{*}$, a contradiction.

Theorem 2.4. For any connected graph $G$ of order $n \geq 3$, and for any two adjacent vertices $u$ and $\mathrm{v}$, where $\operatorname{deg}(\mathrm{u}) \geq 2$ and $\operatorname{deg}(\mathrm{v}) \geq 2, \quad \operatorname{sd} \gamma \operatorname{le}(\mathrm{G}) \leq \operatorname{deg}(\mathrm{u})+\operatorname{deg}(\mathrm{v})-|\mathrm{N}(\mathrm{u}) \cap \mathrm{N}(\mathrm{v})|-1$.

Proof. We may assume that $\mathrm{N}(\mathrm{u}) \cap \mathrm{N}(\mathrm{v}) \neq \varphi$ for otherwise our result follows from theorem2.3.let $\mathrm{N}(\mathrm{v})=\{\mathrm{v} 1, \mathrm{v} 2, \ldots \mathrm{vk}\}$ where $\mathrm{u}=\mathrm{v} 1$ and if $\mathrm{N}(\mathrm{u}) \cap \mathrm{N}[\mathrm{v}] \neq \varphi$. Let $\mathrm{N}(\mathrm{u}) \cap \mathrm{N}[\mathrm{v}]=\left\{\mathrm{u}_{1}, \mathrm{u}_{2}, \ldots \mathrm{ut}\right\}$. Let $\mathrm{G}^{\prime}$ 
be the graph obtained by subdividing the edge vvi with subdivision vertex $\mathrm{x}_{\mathrm{i}}$, for $1 \leq \mathrm{i} \leq \mathrm{k}$, and the edge uuj for $1 \leq \mathrm{j} \leq \mathrm{t}$. Let $A$ be the set of the subdivision vertices and $D^{\prime}$ a $\gamma$ le $\left(G^{\prime}\right)$-set. Clearly no vertex of $G$ locating equitable dominates $v$ in $G^{\prime}$, and so $\left|D^{\prime} \cap A\right| \geq 1$. We Show that $\gamma$ le $\left(G^{\prime}\right)>$ $\gamma$ le $(G)$. IT suffices for us to show that $\gamma_{\mathrm{le}}(G) \leq\left|\mathrm{D}^{\prime}\right|-1$, since then $\gamma \mathrm{le}_{\mathrm{e}}\left(\mathrm{G}^{\prime}\right)=\left|\mathrm{D}^{\prime}\right| \geq \gamma_{\mathrm{le}}(\mathrm{G})+1$. One of $\mathrm{u}$ or $\mathrm{v}$ must be in $\mathrm{D}^{\prime}$ to locating equitable dominate $\mathrm{x} 1$. If both $\mathrm{u}$ and $\mathrm{v}$ are in $\mathrm{D}^{\prime}$, then $\mathrm{D}^{\prime}-\mathrm{A}$ is $\mathrm{a}$ LEDS of $G$, and so $\gamma l e(G) \leq\left|D^{\prime}-A\right| \leq\left|D^{\prime}\right|-1$ Assume $u \in D^{\prime}$ and $v \notin D^{\prime}$. Then every neighbor of $v$ in $\mathrm{G}$ is in $\mathrm{D}^{\prime}$ to locating equitable dominate $\{\mathrm{x} 1, \mathrm{x} 2, \ldots \mathrm{xk}\}$ and some $\mathrm{xi}$ is in $\mathrm{D}^{\prime}$ to dominate $\mathrm{v}$. If $\left|D^{\prime} \cap A\right| \geq 2$, then ( $\left.D^{\prime}-A\right) \cup\{v\}$ is a locating equitable dominating setof $G$, and so $\gamma l e(G) \leq \mid D^{\prime}-$ $A|+1 \leq| D^{\prime} \mid-1$. On the other hand, if $\left|D^{\prime} \cap A\right|=1$, then since $D^{\prime}-\{u\}$ locating equitable dominates $\mathrm{N}(\mathrm{u})-\mathrm{N}[\mathrm{v}]$, it follows that (D'-A- $\{\mathrm{u}\}) \cup\{\mathrm{v}\}$ is a LEDS of $\mathrm{G}$ and so $\gamma \mathrm{le}(\mathrm{G}) \leq\left|\mathrm{D}^{\prime}-\mathrm{A}\right| \leq\left|\mathrm{D}^{\prime}\right|$ 1.Assume $v \in D^{\prime}$ and $u \notin D^{\prime}$. If $\left|D^{\prime} \cap A\right| \geq 2$, then ( $\left.D^{\prime}-A\right) \cup\{u\}$ is a locating equitable dominating set of $G$, and so $\gamma \operatorname{le}(G) \leq\left|D^{\prime}-A\right|+1 \leq\left|D^{\prime}\right|-1$. Therefore, assume that $\left|D^{\prime} \cap A\right|=1$. The vertex of $A$ in $D^{\prime}$ is a neighbor of $v$ in $G^{\prime}$. If $x 1 \in D^{\prime}$, then all neighbors of $v$ in $G$, except for possible $u$, are locating equitably dominated by $D^{\prime}-\left\{v_{1}, x_{1}\right\}$. Thus, $\left(D^{\prime}-\left\{v_{,} x_{1}\right\} \cup\left\{v_{i}\right\}\right.$ for some vertex vi $\in N$ $(\mathrm{u}) \cap \mathrm{N}(\mathrm{v})$ is a locating equitable dominating set of $\mathrm{G}$, and so $\gamma \mathrm{le}(\mathrm{G}) \leq\left|\mathrm{D}^{\prime}\right|-1$. Therefore, we assume that $\mathrm{x} 1 \notin \mathrm{D}$ '. But then some vi $\in \mathrm{NG}_{\mathrm{G}}(\mathrm{v}) \cap \mathrm{NG}_{\mathrm{G}}(\mathrm{u})$ must be in $\mathrm{D}^{\prime}$ to locating equitably dominate $\mathrm{u}$, whence $D^{\prime}-A$ is a locating equitable dominating set of $G$ and $\gamma l e(G) \leq\left|D^{\prime}\right|-1$.

\section{Locating Equitable Independence Subdivision numbers.}

The independence subdivision number of any graph is either one or two. We then characterize the class of graphs having independence subdivision number two.

If $\operatorname{sd} \beta$ le $(G)=1$, for some graph $G=(V, E)$, then , by definition, there must exist an edge uv $\in E$, which when subdivided into edges $u x$ and $x v$ results in a graph $G^{\prime}$ for which $\beta$ le $\left(G^{\prime}\right)$ $=\beta 1 \mathrm{e}(\mathrm{G})+1$. This can happen in only one of two ways, either $G$ has a $\beta$ le $(G)-$ set which does not contain either $u$ or $v$, or $u v$ is a pendant edge and $G$ has a $\beta l e(G)$ - set $D$ which contains the support vertex $\mathrm{u}$ but not the leaf $\mathrm{v}$, in which case $\mathrm{D} \cup\{\mathrm{v}\}$ becomes a larger independent set when the edge $u v$ is subdivided into $\mathrm{ux}$ and $\mathrm{xv}$.

Proposition 3.1. For every graph $G$ having a $\beta$ le $(G)$ - set $D$, where the subgraph G[V-D] induced by V-D has at least one edge, $\operatorname{sd} \beta l e(G)=1$.

Corollary 3.2. For every graph $\mathrm{G}$ having an odd cycle, $\operatorname{sd} \beta l e(G)=1$.

Proposition 3.3. For every graph $G$ having a $\beta$ le $(G)$ - set $D$ and a pendant edge uv, where $D$ contains the support vertex $\mathrm{u}$ ( and not the leafv ), $\operatorname{sd} \beta$ le $(\mathrm{G})=1$.

Proposition 3.4. For every graph $G$ having a $\beta l e(G)$ - set $D$, and a vertex $u \in D$ which is adjacent to at least two vertices in $\mathrm{V}-\mathrm{D}, \operatorname{sd} \beta$ le $(\mathrm{G}) \leq 2$.

Corollary 3.5. For every graph $\mathrm{G}$ having an even cycle, $\operatorname{sd} \beta l e(G) \leq 2$.

Proposition 3.6. For any star $\mathrm{K}_{1}, \mathrm{~m}, \quad \operatorname{sd} \beta$ le $\left(\mathrm{K}_{1}, \mathrm{~m}\right)=\mathrm{m}$.

Theorem 3.7. For any connected graph $\mathrm{G}$ of order $\mathrm{n} \geq 3$, either

(i) $\mathrm{G}=\mathrm{K} 1, \mathrm{~m}$ and $\operatorname{sd} \beta \mathrm{le}(\mathrm{G})=\mathrm{m}$, or

(ii) $1 \leq \operatorname{sd} \beta l e(G) \leq 2$.

Proof. Assume first that $\mathrm{G}$ is connected and contains a cycle. By corollary 4 , if $\mathrm{G}$ contains an odd cycle, then $\operatorname{sd} \beta l e(G)=1$.

If $\mathrm{G}$ has no odd cycle, then it must have a even cycle. By corollary 7 , we can conclude that $\operatorname{sd} \beta 1 \mathrm{e}(\mathrm{G}) \leq 2$.

Assume therefore that $\mathrm{T} \neq \mathrm{K} 1, \mathrm{~m}$, for $\mathrm{m} \geq 3$, and hence that the diameter of $\mathrm{T}$ is at least three.

Case 1. If $\mathrm{T}$ has a $\beta$ le $(\mathrm{T})$-set $\mathrm{D}$, for which $\mathrm{G}[\mathrm{V}-\mathrm{D}]$ has an edge, then by Proposition 3.1, $\operatorname{sd} \beta$ le $(G)=1$. 
Case 2. For every $\beta$ le $(T)-$ set $D, V-D$ is an independent set.Let $D$ be any $\beta$ le $(T)-$ set.Since $T$ is connected, and has diameter at least three, there must be at least one vertex in $\mathrm{D}$ which is adjacent to two or more vertices in V-D. By Proposotion6 it then follows that $\operatorname{sd} \beta$ le $(G) \leq 2$.

It follows from the previous theorem that every connected graph of order $n \geq 3$ can be placed into one of three classes, according to their independence subdivision number:

Class I : Graphs G for which $\operatorname{sd} \beta \operatorname{le}(\mathrm{G})=1$.

Class II : Graphs G for which sd $\beta 1 \mathrm{e}(\mathrm{G})=2$.

Class III: Graphs $\mathrm{G}=\mathrm{K} 1, \mathrm{~m}$ for $\mathrm{m} \geq 3$.

It follows from Corollary 3.2 that class I contains all graphs which are not bipartite. Class I also contain some bipartite graphs G,i.e., those having a $\beta l e(G)$ - set $D$, for which the induced subgraph G[V-D] contains at least one edge or those having a $\beta$ le $(\mathrm{G})$ set which includes at least one support vertex. Class II, which consists of all graphs $G$ for which $\operatorname{sd} \beta \operatorname{le}(G)=2$, contains only bipartite graphs,eg.,C4, for every $\beta$ le $(G)$ - set $D$ of which, V-D is an independent set. This class includes, for example, all even cycles $\mathrm{C}_{2} \mathrm{k}$, all odd paths $\mathrm{P}_{2} \mathrm{k}+1$, and all complete bipartite graphs $\mathrm{K}_{\mathrm{r}, \mathrm{s}}, 2 \leq \mathrm{r} \leq \mathrm{s}$.

Theorem 3.8. A connected graph $G$ is in Class II if and only if either $G=K 1,2$ or $G$ is bipartite with partite sets $\mathrm{V}_{1}$ and $\mathrm{V}_{2}$ such that either

(a) $2 \leq\left|V_{1}\right|=\left|V_{2}\right|=\beta$ le $(G)$, and $V_{1}$ and $V_{2}$ are the only $\beta$ le $(G)$,-sets, or

(b) $2 \leq\left|\mathrm{V}_{1}\right|<\left|\mathrm{V}_{2}\right|=\beta 1 \mathrm{e}(\mathrm{G})$, and $\mathrm{V}_{2}$ is the unique $\beta$ le $(\mathrm{G})$-set.

Proof. If $G=K_{1}, 2$, then the theorem holds. First assume that $G \neq K_{1,2}$ is bipartite with partite sets $V_{1}$ and $V_{2}$ such that either (a) or (b) holds. Since $G$ is connected and not a star.it follows from theorem 3.7, that $1 \leq \operatorname{sd} \beta \operatorname{le}(G) \leq 2$.

We show that $\operatorname{sd} \beta$ le $(G) \neq 1$. Assume to the contrary that subdividing the edge v1v2 yielding v1vv2 for some $\mathrm{v} 1 \in \mathrm{V} 1$ and $\mathrm{v}_{2} \in \mathrm{V}_{2}$ increases the independence number, and let $\mathrm{G}^{\prime}$ be the graph obtain from $G$ by subdividing edge v1v2. If condition (a) holds, then $2 \leq\left|V_{1}\right|=\left|V_{2}\right|=\beta$ le(G) and $V_{1}$ and $\mathrm{V}_{2}$ are the unique $\beta$ le $(\mathrm{G})$-sets. If $\mathrm{x}_{1} \in \mathrm{V}_{1}$ is an endvertex with support $\mathrm{y}_{2} \in \mathrm{V}_{2}$, then $\mathrm{V}_{2}-$ $\left\{\mathrm{y}_{2}\right\} \cup\{\mathrm{x} 1\}$ is another $\beta$ le $(\mathrm{G})$-sets. Similarly, $\mathrm{V}_{2}$ has no endvertices. Thus, $\delta(\mathrm{G}) \geq 2$. but since v1 (respectively,v2) has at least two neighbors in $\mathrm{V}_{2}$ (respectively, $\left.\mathrm{V}_{1}\right)$, it follows that $\beta$ le $\left(\mathrm{G}^{\prime}\right)=$ $\beta$ le $(\mathrm{G})$, contradicting our assumption. If condition (b) holds, then $2 \leq\left|\mathrm{V}_{1}\right|=\left|\mathrm{V}_{2}\right|=\beta$ le $(\mathrm{G})$, and $\mathrm{V}_{2}$ is the unique $\beta$ le $(\mathrm{G})$-sets.Hence for every vertex $\mathrm{u} \in \mathrm{V}_{1}, \operatorname{deg}(\mathrm{u}) \geq 2$, and the result follows as before. Thus, $\mathrm{G} \in \mathrm{ClassII}$.

For the converse, assume that connected graph $\mathrm{G} \neq \mathrm{K} 1,2 \in \mathrm{Class} I$, i.e. $\operatorname{sd} \beta l \mathrm{e}(\mathrm{G})=2$.

Let $\mathrm{D}$ be a $\beta \mathrm{le}(\mathrm{G})$-sets.Proposition 3 implies that $\mathrm{V}-\mathrm{D}$ is independent and hence, $\mathrm{g}$ is bipartite. Since $G$ is connected and not a star, $2 \leq|V-D| \leq|D|=\beta$ le $(G)$. If any vertex,say v,in V-D has exactly one neighbor, say $u$, in $D$, then subdividing the edge uv forming uxv increases the independence number since $\mathrm{D} \cup\{\mathrm{v}\}$ is an independent set, contradicting that $\mathrm{G} \in \mathrm{Class}$ II. Thus every vertex in V-D has at least two neighbors in D. Note that if $|\mathrm{D}|=|\mathrm{V}-\mathrm{D}|$, then both $\mathrm{d}$ and V-D are $\beta$ le $(G)$-sets implying that $\delta(G) \geq 2$.Suppose $D^{\prime}$ is a $\beta$ le $(G)$-sets that is not a partite set of $G$, that is, $D^{\prime} \cap D=A \neq \varphi$ and $D^{\prime} \cap(V-D)=B \neq \varphi$. Let $C=D-A$ and $F=V-D-B$. Note that $D^{\prime}=A \cup B$ and $V-D^{\prime}=(D-A) \cup(V-D-B)=C \cup F$.If $C \cup F$ contains an edge, then by Proposition 3.1. $\operatorname{sd} \beta l e(G)=1$. But since $G \in$ ClassII, $C \cup F$ must be an independent set. But in this case there are no edge between $A \cup F$ and $C \cup B$, implying that $G$ is not a connected graph, a contradiction. Hence, either condition (a) or (b) holds. 


\section{References}

[1] J.A.Bondy and U.S.R.Murty, Graph Theory with Applications (American Elsevier, New York, 1977).

[2] G.Chartant and L.Lesniak,Graphs \& Digraphs (Wadsworth and Brooks/cole, Monterey, CA, third edition, 1996).

[3] F. Harary, Graph theory (Addison-Wesley,Reading,MA,1969).

[4] T.W.Haynes, S.T.Hedetniemi and P.J. Slater Fundamentals of Domonation in Graphs (Marcel dekker, Inc., New York, 1998).

[5] T.W.Haynes, S.T.Hedetniemi and P.J.Slater, Domonation in Graphs Advanced topics (Marcel dekker, Inc., New York, 1998).

[6] D.B. West, Introduction to Graph Theory (Prentice Hall, New Jersey, 1996). 